\title{
Subretinal Bone Formation in a Patient with Giant Cell Tumor of the Sphenoid
}

\author{
Christopher K. Hwang ${ }^{a}$ George Baker Hubbard III ${ }^{a} \quad$ Louis Rapkin $^{b}$ \\ Hans E. Grossniklaus a, c \\ Departments of a Ophthalmology, ${ }^{b}$ Pediatrics and ${ }^{\mathrm{C}}$ Pathology, Emory University School of \\ Medicine, Atlanta, Ga., USA
}

\section{Key Words}

Subretinal bone formation - Giant cell tumor of the sphenoid · Denosumab

\begin{abstract}
Background/Aims: To report a case of subretinal bone formation after the treatment with denosumab for a giant cell tumor of the sphenoid, which had recurred after surgical resection. Methods: The clinical history and fundus findings including imaging, histologic and immunohistochemical features of the primary tumor and subretinal lesion were reviewed. Results: A 14-year-old boy was evaluated for a suprasellar mass. Resection of the lesion showed giant cell tumor of bone (GCT). An MRI study at the 1-month follow-up appointment showed tumor progression, and denosumab was initiated. Two months after the initial presentation, the patient developed a worsening scotoma of the right eye. Dilated fundus examination showed a yellow-tan-colored subretinal mass temporal to the fovea. The subretinal lesion was removed and showed lamellar bone with associated fibrocellular tissue. Conclusion: Denosumab therapy for GCT of the sphenoid may be associated with subretinal bone formation.
\end{abstract}

(C) 2014 S. Karger AG, Base

Giant cell tumor of bone (GCT) is usually a benign tumor that occurs spontaneously in the epiphysis or metaphysis of a long bone [1]. GCT is locally destructive, and thus surgical resection is the current treatment of choice [1]. GCT in the cranium is rare, and pulmonary metastasis occurs in less than $5 \%$ of patients [2]. 
Ocular Oncology

and Pathology

Hwang et al.: Subretinal Bone Formation in a Patient with Giant Cell Tumor of the Sphenoid
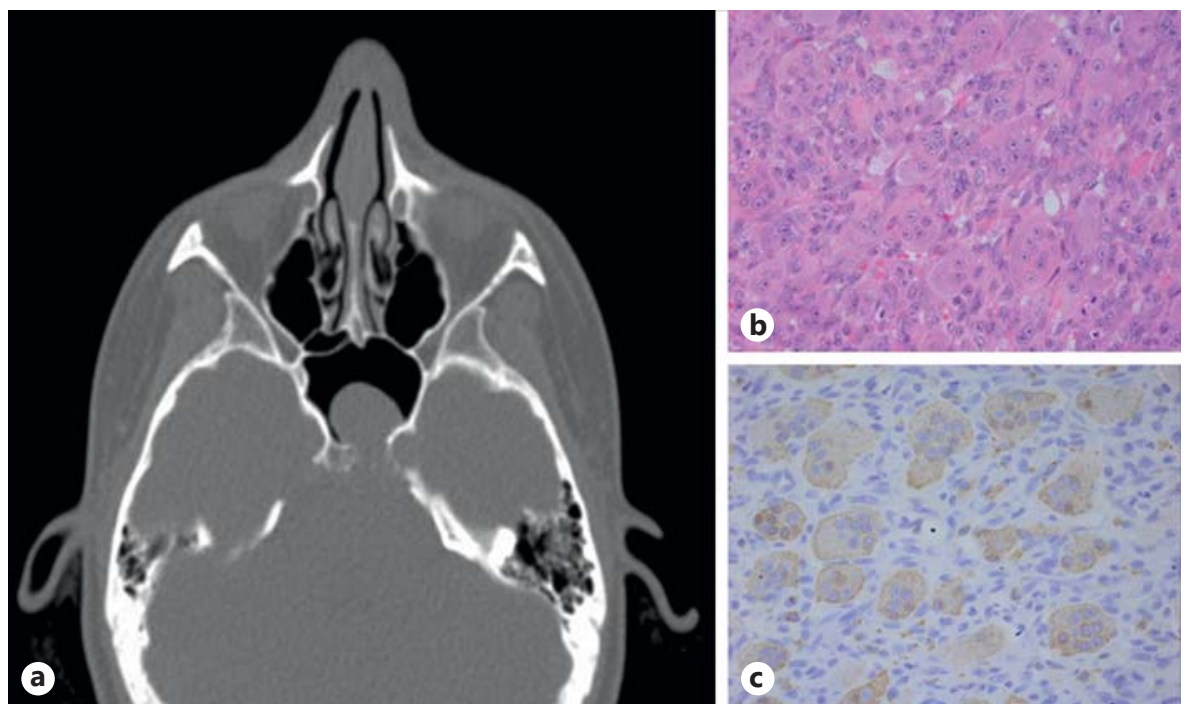

Fig. 1. CT and microscopic images of GCT of the sphenoid. a Head CT showed a lobulated mass at the superior aspect of the clivus. b Microscopic examination of the specimen showed numerous evenly distributed multinucleated giant cells surrounded by fibrovascular tissue interspersed with mononuclear cells. HE. $\times 100$. c Immunohistochemical stain of the giant cells was positive for CD68. $\times 100$.

We report a case of subretinal bone formation in a patient who was treated with denosumab for GCT of the sphenoid and discuss the possibility of the subretinal mass arising through a reactive process, which is defined as benign bone with osteoblastic and osteoclastic activity precipitated by denosumab.

\section{Case Report}

A previously healthy 14-year-old Caucasian boy presented to the emergency department with a 1-week history of headache, diplopia, dizziness, nausea, and vomiting. He played high school football but denied any history of head trauma. His physical examination revealed a left sixth nerve palsy.

A head CT imaging study revealed a hyperdense suprasellar mass more prominent on the left and associated with significant bone destruction (fig. 1a). A follow-up head MRI study showed a lobulated mass at the superior aspect of the clivus extending into the sphenoid sinus as well as the posterior clinoids, causing a significant bone destruction. The mass was contiguous, approximately three quarters circumferential to the left internal carotid artery, and measured $2.7 \times 2.3 \times 2.7 \mathrm{~cm}$. Post-contrast images demonstrated minimal enhancement. No other abnormalities were described on the MRI, although on review there was a small hyperdense area in the posterior sclera/choroid in the right eye. CT imaging studies of the chest, abdomen, and pelvis showed no evidence of metastatic disease. Laboratory studies for growth hormone, prolactin, thyroid stimulating hormone, free T4, luteinizing hormone, follicular stimulating hormone, beta-human chorionic gonadotropin, alpha-fetoprotein, and carcinoembryonic antigen levels were all within normal limits.

Sublabial transsphenoidal resection of the sphenoid mass was performed, resecting approximately $60-70 \%$ of the mass. Microscopic examination of the specimen (fig. 1b) showed a tumor composed of numerous evenly distributed multinucleated giant cells and a background of fibrovascular tissue infiltrated with mononuclear cells. These cells had mildly pleomorphic, vesiculated nuclei and prominent nucleoli. Immunohistochemical stains were positive for CD68 in the giant cells (fig. 1c). Subsequently the diagnosis of GCT was made.

At his 1-month follow-up, the patient showed symptoms similar to those observed at his original presentation. An MRI showed tumor progression with almost complete regrowth of the tumor. Dexamethasone was restarted for symptomatic control of increased intracranial pressure. Treatment options reviewed at that 
Ocular Oncology

and Pathology

Hwang et al.: Subretinal Bone Formation in a Patient with Giant Cell Tumor of the Sphenoid
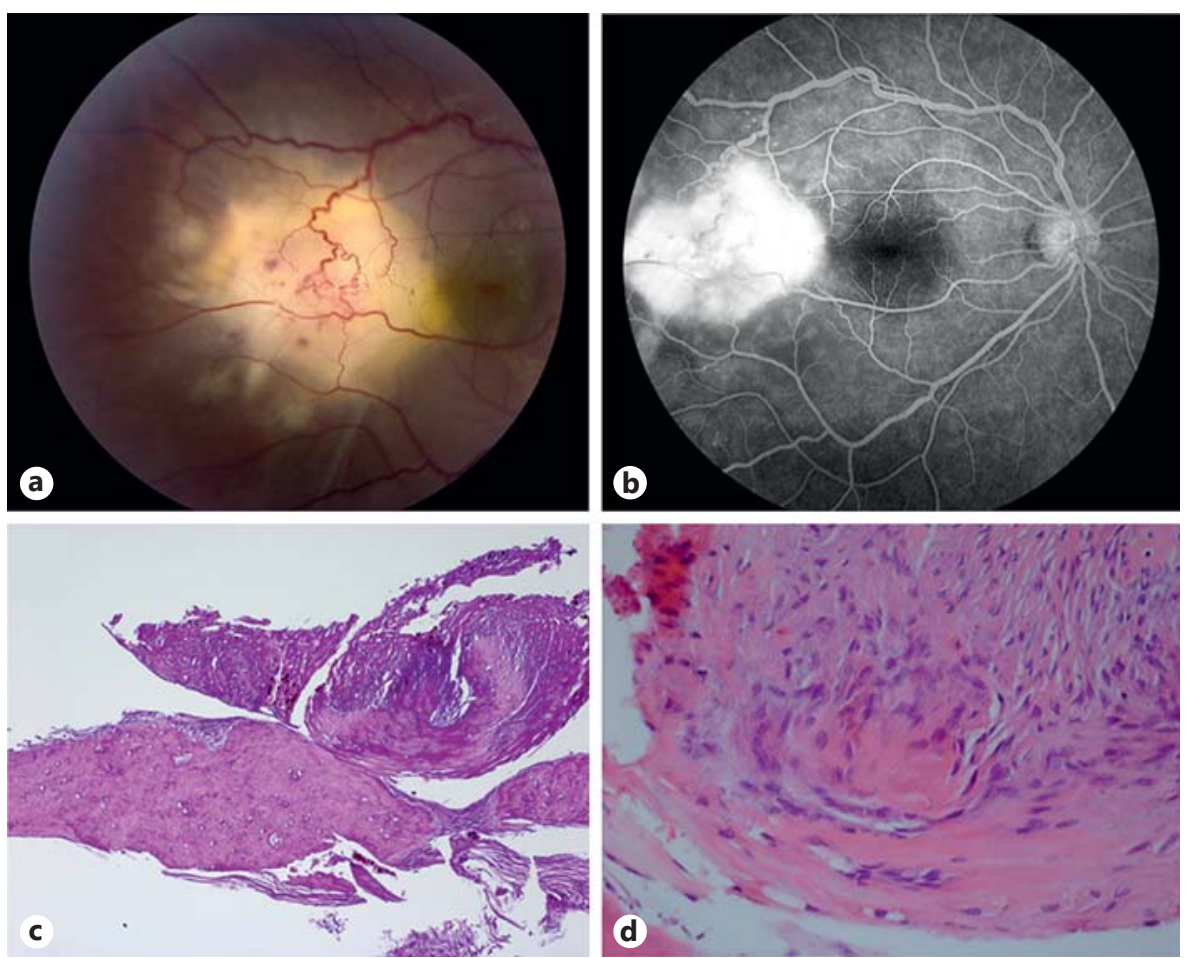

Fig. 2. Fundus findings and histologic features of the subretinal mass. a The subretinal mass initially appeared to be a chorioretinal scar. b Fluorescein angiography of the eye showed early hyperfluorescence of the subretinal mass. c The retinal mass, which was comprised of lamellar bone surrounded by osteoclasts and fibrocellular tissue, was positive for periodic acid-Schiff stain. $\times 25$. $\mathbf{d}$ Rare giant cells were present in the specimen. HE. $\times 100$.

time included radiotherapy or denosumab. The family decided to treat the tumor with denosumab (120-mg subcutaneous injection every 28 days), given the likelihood of panhypopituitarism with radiotherapy. Two months after the initial presentation, the diplopia had resolved completely and imaging documented tumor regression. Serial visual field tests, however, showed a worsening scotoma in the right eye, and 4 months after the initial presentation, the boy's visual acuity in the right eye worsened.

Examination showed that the visual acuity was 20/80 in the right eye and 20/20 in the left eye. No relative afferent pupillary defect was present, and ocular motility was full. The slit-lamp examination revealed a $1+$ cell in the anterior vitreous in the right eye but no cell in the left eye. The dilated fundus examination revealed a yellow-tan-colored subretinal mass temporal to the fovea in the right eye (fig. 2a). There were strands of subretinal material emanating inferiorly and temporally from the main lesion. Overlying the lesion was extensive vascular remodeling of the retinal vasculature. There was yellow exudate and subretinal fluid nasal to the lesion underneath the central fovea. No additional lesions in the periphery were seen. On the left, the disk, macula vessels, and periphery were all normal with the exception of a mild disc pallor.

Fluorescein angiography of the right eye showed early hyperfluorescence of the subretinal lesion with leakage in the late frames (fig. 2b). OCT images showed subretinal fluid in the macula on the right. Serologic studies for Toxocara and toxoplasmosis, QuantiFERON for tuberculosis, and rapid plasma reagin study for syphilis were all negative. An injection of periocular Kenalog did not result in improvement.

The right subretinal lesion was extracted and submitted for histologic analysis. Microscopic examination of the toluidine blue-stained thick section showed lamellar bone surrounded by osteoclasts and fibrocellular tissue, and the specimen was positive for periodic acid-Schiff stain (fig. 2c). The fibrocellular tissue present in the specimen was composed of spindle-shaped cells, lymphocytes, histiocytes, and extracellular collagen fibrils. There were rare osteoclasts at the edge of the lesion (fig. $2 \mathrm{~d}$ ). The lesion was interpreted to represent lamellar bone with fibrocellular tissue consistent with a reactive subretinal bone formation. 
Ocular Oncology

and Pathology

\begin{tabular}{l|l}
\hline Ocul Oncol Pathol 2015;1:34-38 \\
\hline DOI: 10.1159/000366560 & $\begin{array}{l}\text { ( 2)14 S. Karger AG, Basel } \\
\text { www.karger.com/oop }\end{array}$ \\
\hline
\end{tabular}

Hwang et al.: Subretinal Bone Formation in a Patient with Giant Cell Tumor of the Sphenoid

During the follow-up period, the sphenoid tumor continued to shrink in the first 9 months of therapy and remained stable after that time. There has been no evidence of metastasis. Three months after vitrectomy for removal of the subretinal mass, the patient developed proliferative vitreoretinopathy with retinal detachment requiring scleral buckle, repeat vitrectomy, lensectomy, and silicone oil tamponade.

\section{Discussion}

GCT is a tumor of low metastatic potential that occurs spontaneously and almost exclusively in the epiphysis or metaphysis of a long bone [2]. It generally occurs in patients aged 20-40 years, more commonly in females than males, and very rarely in children and adolescents [2]. GCT comprises $3-5 \%$ of all tumors, $15-20 \%$ of benign bone tumors [3], and rarely originates in the cranium [3-5]. However, when it does, it appears to originate preferentially in the sphenoid and temporal bones [4]. Metastasis rarely occurs $(<5 \%)$, but when it does, it usually occurs in the lungs [3]. GCT is locally destructive, and thus surgical resection is the current treatment of choice [1]. Additionally, radiation therapy has been successfully used, and denosumab, which is a monoclonal antibody against receptor activator of nuclear factor- $\kappa \mathrm{B}$ ligand (RANKL), was recently FDA approved for the treatment of unresectable and progressive GCT.

The origin of our patient's subretinal bone is uncertain. It is possible that the subretinal bone mass was derived from the primary GCT of the sphenoid, but GCT metastasis to the retina has never been described. GCT metastases occur significantly more frequently in patients in whom surgical procedures are performed to remove the tumor and in patients with GCT recurring locally [4]. It is possible that the GCT of the sphenoid could have metastasized to the choroid in a similar fashion. In our patient, the subretinal lesion was not clinically detected until after the tumor was surgically resected, although a hyperdense area in the posterior aspect of the eye had been noted on MRI prior to treatment.

Nevertheless, the subretinal mass more likely represents a reactive bone formation involving a pre-existing lesion such as an occult choroidal osteoma or choroidal embolism of lamellar bone fragments, which - though rarely - has been reported to occur in other organs following trauma to the bone including trauma as a result of surgeries [6-8]. Denosumab is a RANKL antagonist that has been shown to suppress bone destruction in different lytic bone tumors including GCT. However, while denosumab prevents reabsorption of normal bone by osteoclasts, it allows for increased new bone formation by osteoblasts [9]. Therefore, denosumab could theoretically allow a bone fragment to increase its size and mass by inhibiting reabsorption. The timing of symptoms in our patient supports this theory. Just weeks after starting the denosumab therapy, his vision worsened rapidly from 20/20 to 20/80 and the subretinal mass was discovered. Histologically, GCT is comprised of osteoclast-like multinucleated giant cells and mononuclear stromal cells [10]. Microscopically, the subretinal specimen consisted of reactive bone formation and did not resemble GCT despite the rare presence of CD68-positive giant cells. Given that the subretinal lesion more closely resembled reactive bone formation rather than the patient's GCT and that the lesion became symptomatic only after treatment, we think that the subretinal mass arose through a reactive process, possibly in a pre-existing lesion. Patients treated with denosumab for primary GCT should undergo fundus examination since a reactive bony lesion may arise in the choroid.

\section{Acknowledgement}

This work was supported in part by an unrestricted department grant from Research to Prevent Blindness, Inc., and NIH NEI P30 06360. 
Hwang et al.: Subretinal Bone Formation in a Patient with Giant Cell Tumor of the Sphenoid

\section{References}

1 Thomas DM, Desai J: Giant cell tumor of bone; in Rose BD (ed): UpToDate. Waltham, UpToDate, 2014.

2 Raskin KA, Schwab JH, Mankin HJ, Springfield DS, Hornicek FJ: Giant cell tumor of bone. J Am Acad Orthop Surg 2013;21:118-126.

-3 Campanacci M: Bone and Soft Tissue Tumors: Clinical Features, Imaging, Pathology and Treatment. Padua, Piccin Nuova Libraria, 1999.

4 Reid R, Banerjee S, Sciot R: Giant cell tumor; in Fletcher CDM, Unni KK, Mertens F (eds): Pathology and Genetics: Tumours of Soft Tissue and Bone. Lyon, IARC Press, 2002.

5 Bertoni F, Unni KK, Beabout JW, Ebersold MJ: Giant cell tumor of the skull. Cancer. 1992;70:1124-1132.

6 Hara N, Minami T: Diffusive pulmonary embolism with bone fragments during spinal surgery. Br J Anaesth 2006;97:119-120.

7 Jenny-Möbius U, Bruder E, Stallmach T: Recognition and significance of pulmonary bone embolism. Int J Legal Med 1999;112:195-197.

-8 Perello L, Gracia I, Fabregas N: Bone embolism during neurosurgery in sitting position. J Neurosurg Anesthesiol 2013;25:93.

-9 Branstetter DG, Nelson SD, Manivel JC, Blay JY, Chawla S, Thomas DM, Jun S, Jacobs I: Denosumab induces tumor reduction and bone formation in patients with giant-cell tumor of bone. Clin Cancer Res 2012;18:44154424.

10 Dorfman HD, Czerniak B: Bone Tumors. St. Louis, Mosby, 1998. 